\title{
The open loop response analysis of duck wing with trust resistance balance and constant elasticity
}

\author{
Haibo Wang ${ }^{1}$, Aijun Zhou, ${ }^{1}$, Junbo Chu $^{2}$ Tianwei $\mathrm{Li}^{1}$ and Guoqiang Liang ${ }^{3}$ \\ ${ }^{1}$ Department of navigation, Dalian Naval Academy Dalian, 116018, China \\ ${ }^{2}$ Department of missile, Dalian Naval Academy Dalian, 116018, China
}

${ }^{3}$ Department of control engineering, Naval aeronautical and astronautical University Yantai, 264001, China

azajwxy@163.com

Keywords: open loop, stability; resistance; balance; duck wing

\begin{abstract}
A kind of virtual flying condition is assumed that the trust and resistance is the same that can keep the force balance during the flying process of high speed aircraft. The main function of this virtual assumption is that it can the unstable open loop aircraft fly a longer time so more date can be gotten and the duck wing response or effect can be analyzed more clearly. So it is a new method and it is effective and meaningful for the open loop dynamic analysis especially for high speed unstable control objects.
\end{abstract}

\section{Introduction}

The thrust changing sharply causes the pitching rotation moment of hypersonic flight vehicle difficult to balance from open-loop characteristics of canard test simulation graph[1-4]. Thus it leads to the divergence of attack angle. So consider selecting thrust resistance balance mode, studying the open loop characteristic of the canard. The purpose of this test is to extend the time of the simulation. The divergence data of attack angle can be observed for a longer time. The open-loop characteristic of canard has the stable situation, which shows that the cause of the hypersonic aircraft attack angle divergence is the mismatch between the thrust and resistance, or the engine thrust is difficult to precise control. The open-loop characteristics of canard is researched on the special circumstances of resistance balance based on the U.S. air force announcing hypersonic vehicle longitudinal model[5-9], in order to have a further insight into superb control complexity problem.

\section{Model Description}

Considering the elastic shape structure, a kind of pitch channel hypersonic aircraft model built according to Lagrange equation is released by USA air force as followed:

$$
\begin{array}{r}
\dot{V}=\frac{T \cos \alpha-D}{m}-g \sin \gamma \\
\dot{\phi}=-2 \varsigma \omega_{n} \phi-\omega_{n}^{2} \phi+\omega_{n}^{2} \phi_{c} \\
\dot{\gamma}=\frac{L+T \sin \alpha}{m V}-\frac{g \cos \gamma}{V} \\
\dot{\alpha}=q-\dot{\gamma} \\
\dot{q}=\frac{M}{I} \\
\dot{h}=V \sin \gamma \\
\ddot{\eta}_{i}=-2 \varepsilon_{m} \omega_{m i} \dot{\eta}_{i}-\omega_{m i}^{2} \eta_{i}+N_{i}
\end{array}
$$


And $V$ is speed, $\gamma_{\text {is }}$ the speed angle, $\alpha$ is attack angle, $Q_{\text {is the attitude angle speed, }} h_{\text {is the }}$ height. $\phi$ is the oil supplying factor, $\delta_{c}$ is the duck wing and $\delta_{e}$ is the lift rudder.

\section{Simulation settings of free flying}

In order to testify the rightness of the model of hypersonic aircraft, choose a initial height as $h_{0}=85000 * 0.3048$, initial speed as $V_{0}=7846 * 0.3048$, initial attack angle as $\alpha_{0}=0.0174$, and other initial state as: $\gamma_{0}=0, q_{0}=0, \eta_{10}=0.4588 * 0.3048 * 14.59, \eta_{20}=-0.08726 * 0.3048 * 14.59$, $\eta_{30}=-0.03671 * 0.3048 * 14.59$, Setting the throttle opening of engine is the switching control law for the PID control law and diffusion control law, and selection

$$
\phi_{c}=k_{e t} e_{v}+k_{s e t} e_{v}+k_{d e} \dot{e}_{v}+k_{e t b} \frac{e_{v}}{\left|e_{v}\right|+\varepsilon_{e v}}, e_{v}>0, \phi_{c}=-300, e_{v}<0
$$

Among them, $e_{v}=V^{d}-V, k_{e t}, k_{\text {set }} 、 k_{d e}, k_{e t b}$ and $\varepsilon_{e v}$ are for the adjustable positive number. The expectations speed is selected as $V^{d}=2391$, control volume is selected as $\delta_{c}=1 / 57.3$, $\delta_{e}=0$, the canard open loop dynamic characteristics of testing superb craft, setting $\dot{\eta}_{i}=0$, setting the flight time as 0.5 seconds.

\section{Numerical Simulation and result analysis}

With above model and air coefficients and set initial condition as above paragraph, write a program with $\mathrm{m}$ language in Matlab software, then the simulation can be done and simulation results can be shown as following figures.

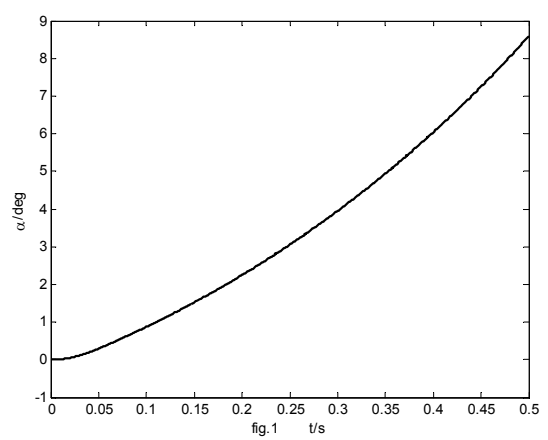

Fig 1 The curve of attack angle

When push resistance is imbalance, the divergence of attack angle is within $0.5 \mathrm{~s}$ to 8.5 degrees, but when the push resistance is balance, the divergence speed reduces to 3.5 degrees. It can be seen that control accuracy of engine thrust has great influence for system.

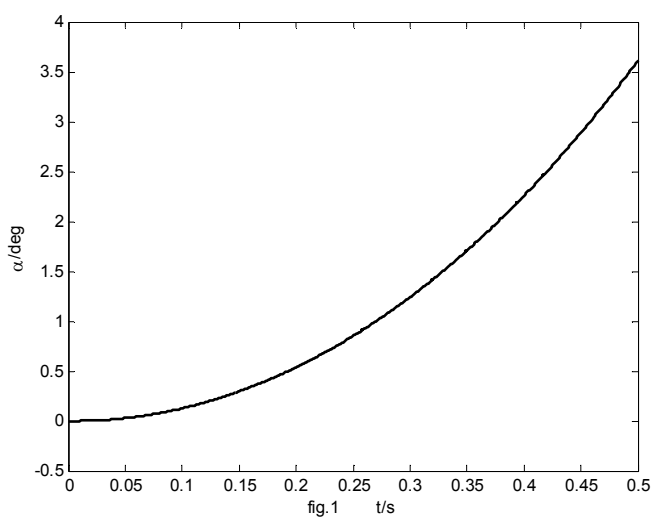

Fig 2 The curve of attack angle

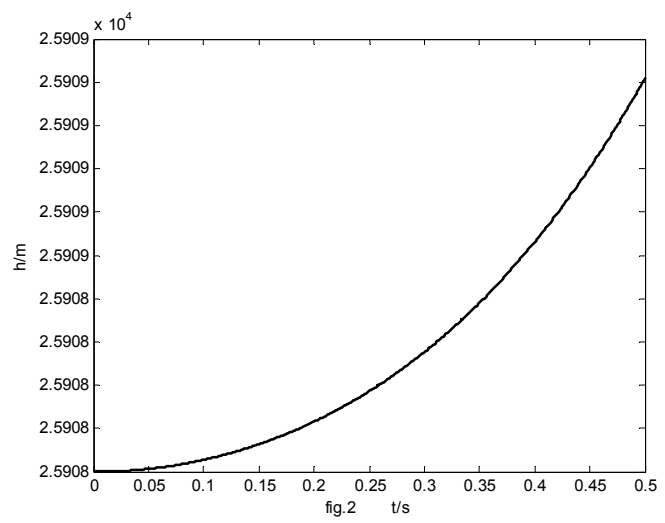

Fig 3 The curve of the height 


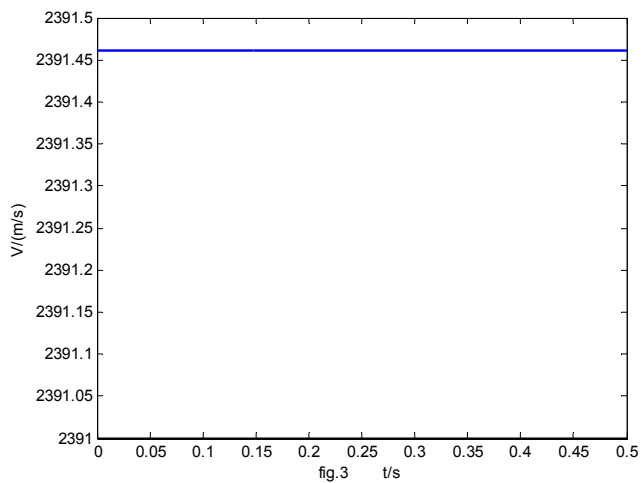

Fig 4 The curve of speed

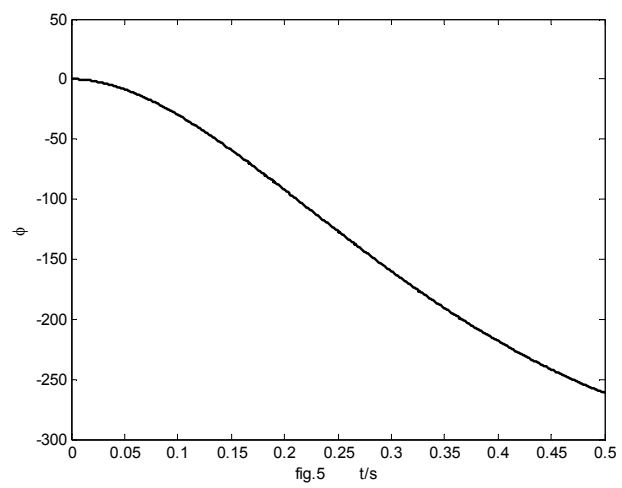

Fig 6 The curve of oil supplying factor

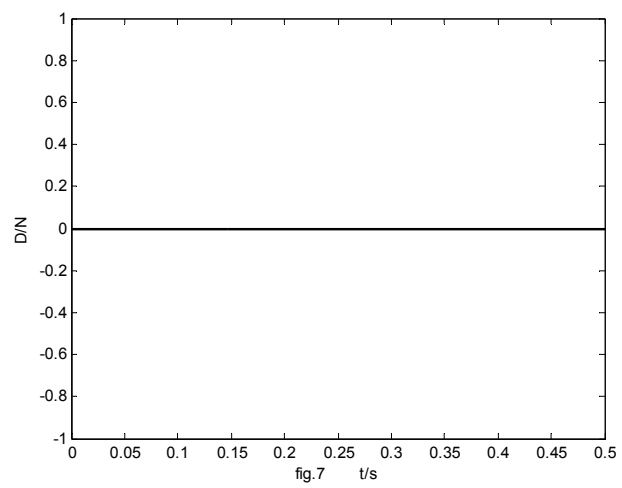

Fig 8 The curve of resistance

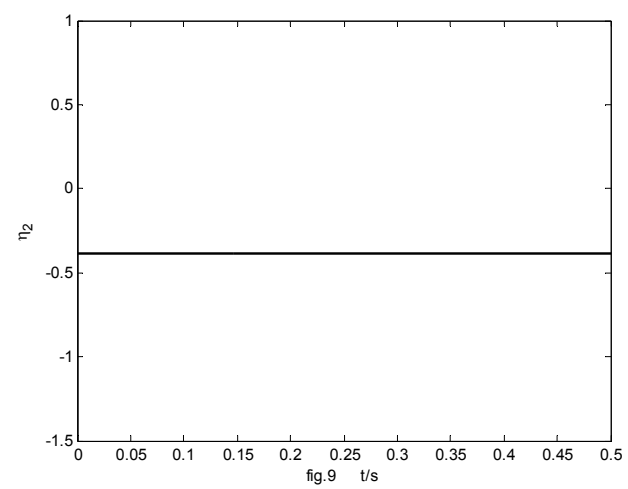

Fig 10 The state of second elastic shape

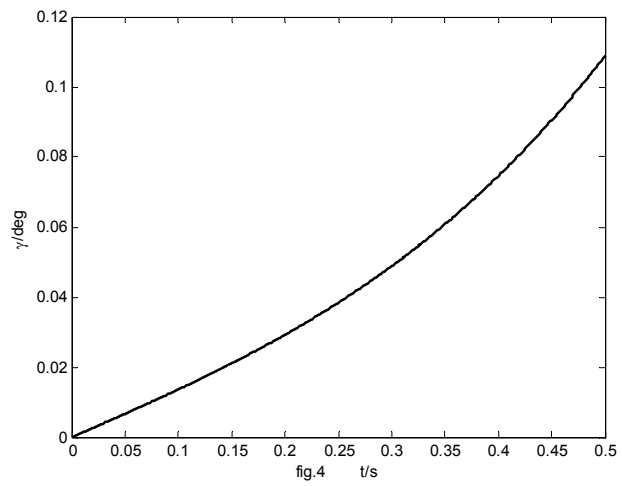

Fig 5 The curve of speed angle

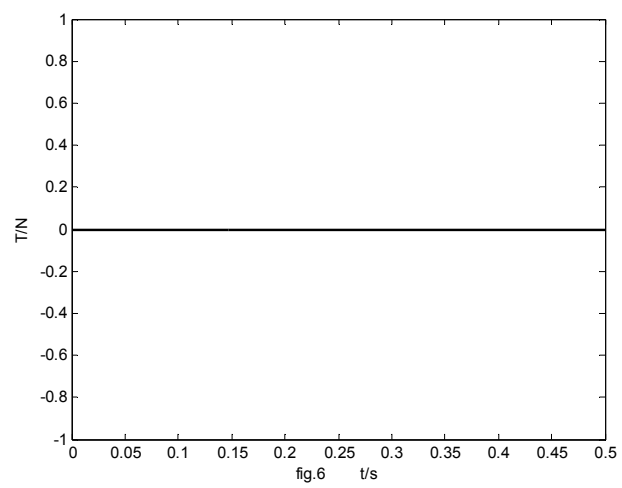

Fig 7 The curve of thrust

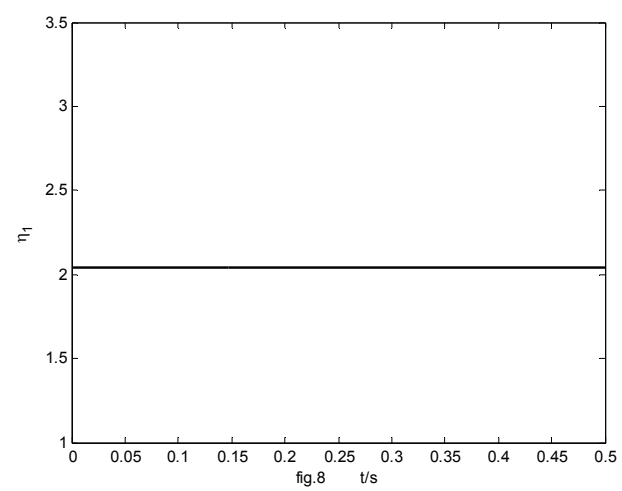

Fig 9 The state of first elastic shape

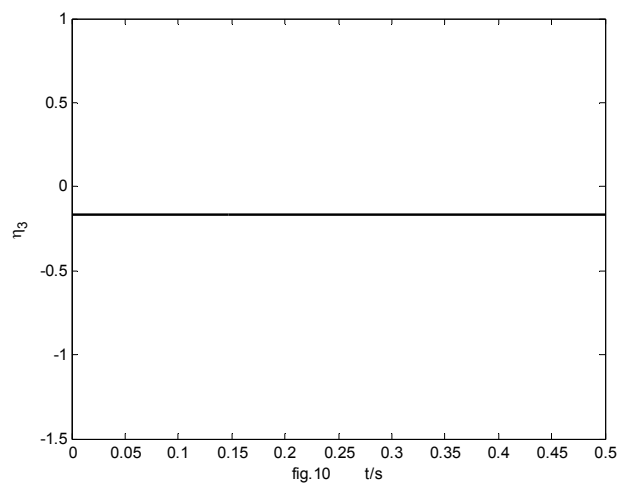

Fig 11 The state of third elastic shape 


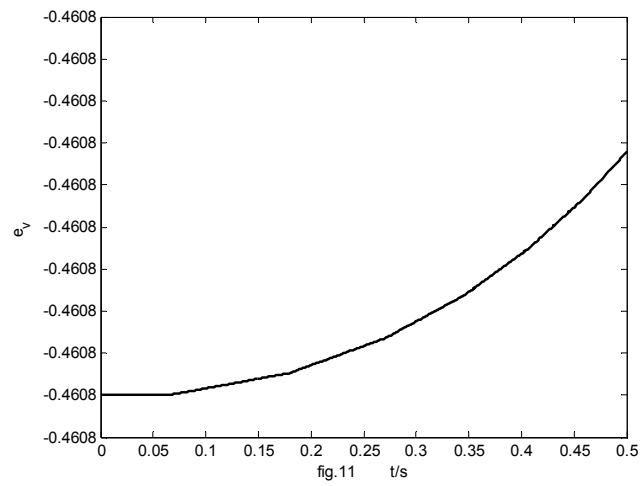

Fig 12 The state of speed error

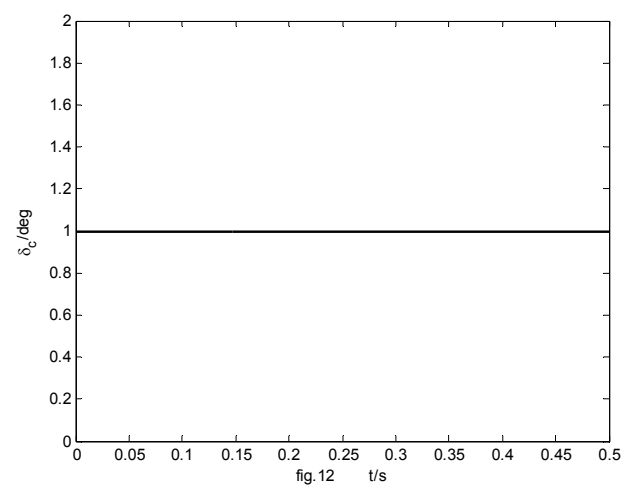

Fig 13 The curve of duck actuator

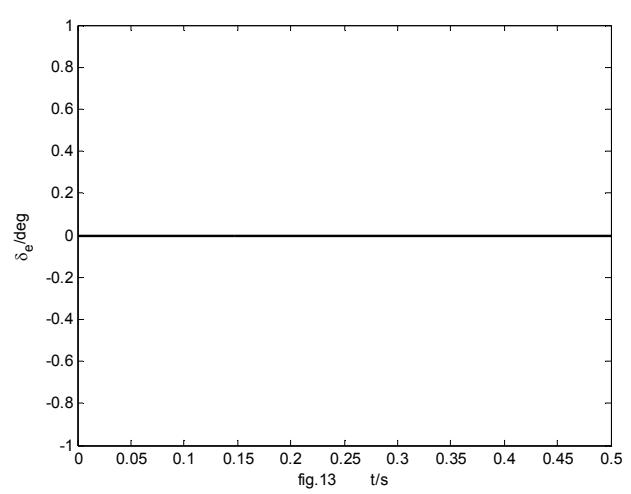

Fig 14 The curve of lift actuator

If you consider to use the way of thrust same with resistance to achieve balance, the simulation results are shown in the figure below:

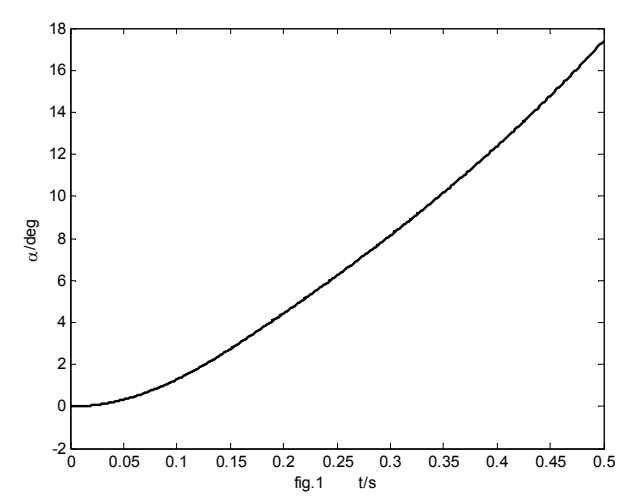

Fig 15 The curve of attack angle

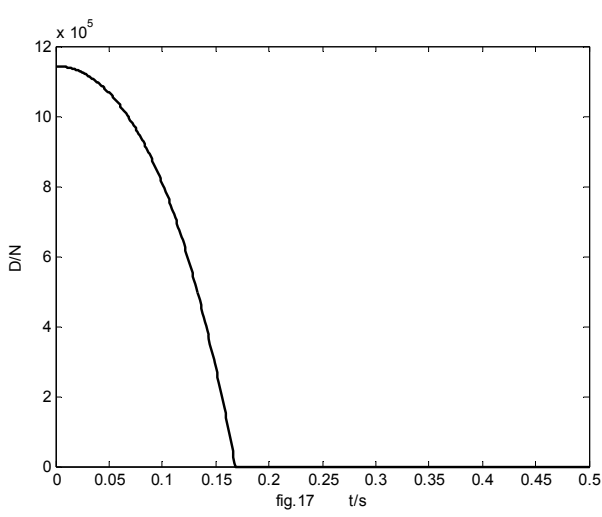

Fig 16 The curve of resistance

The situation of thrust resistance unbalanced is similar to the simulation results, It can be seen that push resistance balance should not the reason that affects the divergence speed fast of attack angle.

\section{Conclusion}

The open loop flight simulation of canard constant is done by setting the hypersonic flight vehicle driving resistance balance with the elastic assumptions in this paper. It illustrates that unstable characteristics of canard to attack angle is not caused by the unbalanced push resistance. By setting three conditions simulation that the thrust resistance is zero, thrust is normal, the resistance is assumed to be equal thrust, thrust resistance is normal, the results show that in addition to the first case that the divergence of attack angle is slow, the other two cases, the divergence of attack angle is almost the same speed. It shows that the divergence of attack angle is caused by the thrust mismatch with resistance torque, rather than the equal of a simple force can guarantee the moment balance. Therefore, considering that the force and moment are match balanced, then the 
unstable image of attack angle can obtain the essence improvement.

\section{References}

[1]Wang J L, Sundararajan N. A nonlinear flight controller design for aircraft[J].Control Eng.Practice, 1995, 3(6): 813-825.

[2]Vaddi S S, Sengupta P. Controller design for hypersonic vehicles accommodating nonlinear state and control constraints, In: AIAA Guidance, Navigation, and Control Conference Chicago, USA, AIAA 2009-6286: $1-19$

[3]Kevin. P. Groves, Andrea. Serranti, Stephen. Yurkovich. Anti-Windup Control for an Air-breathing Hypersonic Vehicle Model. AIAA, 2006-6557: 1-14.

[4]Heller M, Holzapfel F and Sachs G. Robust lateral control of hypersonic vehicles [A]. In: AIAA Guidance, Navigation, and Control Conference and Exhibit [C]. Denver, CO, AIAA 2000-4248.

[5]Chavez F R and Schmidt D K.Flight dynamics and control of elastic hypersonic vehicles-Modeling uncertainties[A].In:AIAA Guidance, Navigation and Control Conference[C]. Washington,DC, AIAA 94-3629

[6]Buschek $\mathrm{H}$ and Calise A J. Fixed order robust control design for hypersonic vehicles[A].In:AIAA Guidance, Navigation and Control Conference [C]. Washington,DC,AIAA 94-3662

[7]M. Ohno, Y. Yamaguchi, T. Hata, M. Takahama, Robust flight control law design for an automatic landing flight experiment [J], Control Engineering Practice, 1999, 7(9):1143-1152

[8]Ohno M, Yamaguchi Y, Hata T, et al. Robust flight control law design for an automatic landing flight experiment [J]. Control Engineering Practice, 1999, 7 (9):1143-1152.

[9]Roy Smith, Asif Ahmed, Robust parametrically varying attitude controller designs for the X-33 vehicle [A], In: AIAA Guidance, Navigation, and Control Conferenceand Exhibit [C], Denver: AIAA, 2000-4158 